\title{
Serum Tumor Necrosis Factor-Alpha as a Competent Biomarker for Evaluation of Disease Activity in Early Rheumatoid Arthritis
}

\author{
Mehreen Inam Illahi ${ }^{1}$, Sofia Amjad ${ }^{1}$, Syed Mehfooz Alam ${ }^{2}$, Syed Tousif Ahmed ${ }^{1}$, Murk Fatima ${ }^{1}$, \\ Moazzam A. Shahid ${ }^{3}$ \\ 1. Physiology, Ziauddin University, Karachi, PAK 2. Rheumatology, Liaquat National Hospital, Karachi, PAK 3. \\ Biochemistry, Ziauddin University, Karachi, PAK
}

Corresponding author: Mehreen Inam Illahi, mehreen.illahi@zu.edu.pk

\section{Abstract}

\section{Aim}

The cytokines particularly tumor necrosis factor-alpha (TNF- $\alpha$ ) have a substantial role in the pathophysiology of rheumatoid arthritis (RA). The goal of this study was to evaluate the role of serum TNF$\alpha$ as a competent biomarker of disease activity in RA and to assess the correlation of serum TNF- $\alpha$ with DAS28-ESR (disease activity score-erythrocyte sedimentation rate in 28 joints) and other markers expressed in serum of RA patients.

\section{Methodology}

The study was conducted from May 2020 to October 2020 after approval from the Ethical Review Committee of Ziauddin University. This cross-sectional study included 90 diagnosed cases of RA from 30 to 65 years with the complaint of arthralgia. Patients from the rheumatology clinic were enrolled in the study by a nonprobability consecutive sampling technique. Informed consent was taken from each patient and they were assessed through a set of questions based upon disability in the performance of daily activities due to RA. Evaluation of serum levels of anti-cyclic citrullinated peptide (ACCP), rheumatoid factor (RF), erythrocyte sedimentation rate, and TNF- $\alpha$ were done by enzyme-linked immunosorbent assay (ELISA). Patients were segregated into groups based upon DAS28-ESR with erythrocyte sedimentation rate as an inflammatory marker. The Kruskal Wallis test was applied for the comparison of different variables in these groups. Spearman correlation was applied for the association between different variables. Multiple variable analysis was performed to assess the predictability of disease activity by serum markers included in the study.

\section{Results}

The results of our study disclosed a significant difference in ACCP, TNF- $\alpha$, tender joint count of 28 joints (TJ-28), swollen joint count of 28 joints (SJ-28), and health assessment questionnaire-disability index (HAQDI) in disease activity groups. A significant correlation of serum TNF- $\alpha$ with DAS28-ESR in RA patients was observed.

Review began 05/18/2021 Review ended 05/22/2021 Published 05/29/2021

\section{() Copyright 2021}

Inam Illahi et al. This is an open access article distributed under the terms of the Creative Commons Attribution License CC-BY 4.0., which permits unrestricted use, distribution, and reproduction in any medium, provided the original author and source are credited.

\section{Conclusion}

This study illustrated a significant correlation of serum TNF- $\alpha$ with DAS28-ESR in RA patients. We found that expression of serum TNF- $\alpha$ may intensify the inflammatory activity in early RA, therefore, RA patients must be screened for this cytokine to monitor that disease activity could be useful for patients undergoing anti-TNF therapy.

Categories: Allergy/Immunology, Rheumatology

Keywords: rheumatoid arthritis, autoimmune disease, erythrocyte sedimentation rate, disease activity score, tumor necrosis factor alpha

\section{Introduction}

Cytokines belong to a diverse family of proteins associated with the inflammatory activity in autoimmune diseases. They portray a significant role in the preservation of homeostasis. An imbalance in the cytokine network leads to enhanced inflammation. Therefore, these cytokines might be valuable as predictive biomarkers of disease activity [1,2]. Cytokines perform an essential role in the cascades that cause articular destruction and the comorbidities associated with immune-mediated joint diseases including rheumatoid arthritis (RA) [3]. RA is a chronic, systemic inflammatory, autoimmune disease that causes deformity and restriction of joint movements [4]. Cytokines are produced by innate immune cells of the synovial membrane in RA patients [3].

Among these cytokines tumor necrosis factor-alpha (TNF- $\alpha$ ) is a principal cytokine, that is chiefly produced 
by macrophages [3]. TNF- $\alpha$ itself acts as a potent inducer of other proinflammatory cytokines and chemokines, thus further enhancing the inflammatory response [5]. In conjunction with other proinflammatory mediators, TNF- $\alpha$ promotes the stimulation of synovial fibroblasts, chondrocytes, and osteoclasts that release tissue-destroying enzymes, the matrix metalloproteinases (MMPs) [6,7]. The MMPs cause degradation of extracellular matrix components thus causing destruction of bone and cartilage which begins very early in the course of RA [8]. Hence, all of these TNF- $\alpha$ activities fuel inflammation in the synovium, increase angiogenesis, promote cartilage and bone resorption [3], suppress regulatory T cells, and promote pain $[9,10]$. TNF- $\alpha$ also amplifies osteoclast activation and differentiation [11]. According to a study, the severity of inflammation was assessed well in RA patients by TNF- $\alpha$ in both pre-treatment and post-treatment phases [7,12].

It has been proposed by many studies that disease activity can be monitored by diagnostic markers for RA, the rheumatoid factor (RF), and anti-cyclic citrullinated peptide (ACPP) [13,14]. However, the levels of these biomarkers do not correlate well with disease activity in RA. There is a growing need to search for new biomarkers to monitor the inflammatory activity in RA and in this context cytokines can be potentially applicative.

In RA, the inflammatory activity can be evaluated by a disease activity score with an erythrocyte sedimentation rate (ESR) as the inflammatory marker (DAS28-ESR) in 28 joints. Evaluation of disease activity guides the clinicians and patients toward a standardized treatment approach. The DAS28-ESR is a widely used measure for the clinical assessment of RA patients. It is a score that includes 28 tender and swollen joint counts, along with a patient global assessment, and a physician global assessment. Values are defined by high disease activity (HAD), moderate disease activity (MDA), low disease activity (LDA), or remission. Improvement or worsening of disease is reflected by changes in score values [15]. The goal of our study was to assess the efficacy of serum TNF- $\alpha$ as a potential biomarker of disease activity in early RA. We evaluated the correlation of serum TNF- $\alpha$ with DAS28-ESR and other markers expressed in serum of RA patients.

\section{Materials And Methods}

This cross-sectional study was conducted from May 2020 to October 2020 after approval from the Ethical Review Committee of Ziauddin University (Refcode: 0920319MIPHY) and included 90 diagnosed cases of RA from 30 to 65 years with the complaint of arthralgia. Patients from the rheumatology clinic were enrolled in this study on the basis of a non-probability consecutive sampling technique. Patients were diagnosed on the basis of 2010 American College of Rheumatology/European League Against Rheumatism (ACR/EULAR) criteria for RA [16]. Study participants were disease-modifying antirheumatic drugs (DMARD) naïve. All participants gave informed consent and filled in a questionnaire about the performance of their activities in daily life as assessed by the Stanford University Health Assessment Questionnaire-disability index (HAQ-DI) [17] which included 20 questions regarding, (1) dressing and grooming, (2) arising, (3) eating, (4) walking, (5) hygiene, (6) reach, (7) grip, and (8) common daily activities. The response of patients were recorded as scores from 0 to 3, mentioned in HAQ-DI: $0=$ able without any difficulty, $1=$ able with some difficulty, $2=$ able with much difficulty, and $3=$ =unable. Clinical examinations were done by a certified trained rheumatologist. The total HAQ score was calculated as given in HAQ-DI. The DAS28 - including 28 tender (TJ28) and swollen joint (SJ28) count, and the ESR - was used to assess clinical disease activity by a DAS28-ESR calculator [18]. Values of DAS28-ESR were defined: HDA (>5.10), MDA (3.21-5.10), LDA (2.61-3.20), or remission (<2.60) [19].

A venous blood sample of $5 \mathrm{ml}$ was taken from each patient by a trained phlebotomist and it was centrifuged at this $3000 \mathrm{rpm}$ to get the serum and stored in multiple aliquots at $-80^{\circ} \mathrm{C}$. RF levels were determined using an RF enzyme-linked immunosorbent assay (ELISA) kit (MBS721682; MyBioSource, San Diego, California, USA). The reference range was from 5.0 to $100 \mathrm{IU} / \mathrm{mL}$, and analytical sensitivity was $1.0 \mathrm{IU} / \mathrm{mL}$. According to the manufacturer's protocol, the assay sample and buffer were incubated together with RF-horseradish peroxidase (RF-HRP) conjugate in a microtiter plate pre-coated with anti-RF antibody for one hour. The wells were washed and incubated with a substrate for the HRP enzyme. A blue-colored complex was formed and later after the addition of stop solution color changed to yellow. The intensity of color was measured spectrophotometrically at $450 \mathrm{~nm}$ in a microplate reader.

ACCP levels were determined using an ACCP antibody, ELISA kit (MBS720363; MyBioSource). The reference range was from 25 to $500 \mathrm{U} / \mathrm{mL}$ and analytical sensitivity of $1.0 \mathrm{U} / \mathrm{ml}$. According to the manufacturer's protocol, the assay sample and buffer were incubated together with CCP-Ab-HRP conjugate in CCP-Ab precoated plate for one hour. The wells were washed and incubated with a substrate for the HRP enzyme. A blue-colored complex of enzyme-substrate reaction was formed. A stop solution was added to terminate the reaction. The intensity of color was measured spectrophotometrically at $450 \mathrm{~nm}$ in a microplate reader.

Assay of serum TNF- $\alpha$ was done by a sandwich ELISA (Cat.No.E0082Hu; Bioassay Technology Laboratory, Shanghai, China). The reference range was from $3 \mathrm{pg} / \mathrm{ml}$ to $900 \mathrm{pg} / \mathrm{ml}$ and analytical sensitivity of 1.52 $\mathrm{pg} / \mathrm{ml}$. According to the manufacturer's protocol, TNF- $\alpha$ present in the sample is added and binds to human TNF- $\alpha$ antibodies coated on the wells. Then, streptavidin-HRP is added and binds to the biotinylated TNF- $\alpha$ antibody. After incubation plate wells were washed. Substrate solution was added and color developed in proportion to the amount of human TNF- $\alpha$. The reaction was terminated by the addition of stop solution 


\section{Cureus}

and absorbance was measured at $450 \mathrm{~nm}$.

\section{Statistical analysis}

Data analysis was done on Statistical Package for Social Sciences (SPSS), version 20 (IBM SPSS Statistics, Armonk, NY). Median and interquartile ranges were calculated for numeric variables The Kruskal Wallis test was applied for comparison of three groups. Spearman's correlation was applied for finding a correlation among the study variables. Multiple variable analysis was performed to assess the predictability of disease activity by serum markers included in the study.

\section{Results}

Table 1 shows metabolic and biochemical variables among three groups of DAS28-ESR in RA patients. The Kruskal Wallis test was applied. Significantly $(p \leqslant 0.001)$ higher levels of serum ACCP were seen in the severe DAS28 group, versus the mild and moderate groups. The TJ-28 was significantly $(\mathrm{p} \leqslant 0.001)$ high in mild and severe DAS-28 groups when compared with the moderate group. A significantly high SJ-28 ( $\leqslant \leqslant 0.001)$ count was seen in the severe DAS28 group than in the mild and moderate groups. The HAQ-DI was significantly $(\mathrm{p}=0.008)$ high in the mild disease activity group than in the severe and moderate sub-groups. The serum TNF- $\alpha$ was significantly ( $\leqslant 0.001$ ) high in severe DAS28 scores versus the moderate and mild disease score groups. Among the three groups of DAS28-ESR, no significant difference was noted in BMI, RF, and ESR values.

\begin{tabular}{|c|c|c|c|c|c|c|c|}
\hline \multirow{2}{*}{ Parameters } & \multicolumn{2}{|c|}{ DAS28-ESR mild (2.61-3.2) } & \multicolumn{2}{|c|}{ DAS28-ESR moderate (3.21-5.1) } & \multicolumn{2}{|c|}{ DAS28-ESR severe $(>5.1)$} & \multirow{2}{*}{ p-Value } \\
\hline & $\mathrm{M}$ & IQR & $\mathrm{M}$ & IQR & $\mathrm{M}$ & IQR & \\
\hline BMI $\left(\mathrm{kg} / \mathrm{m}^{2}\right)$ & 21.6 & 2.6 & 23 & 3.07 & 22.4 & 2.3 & 0.101 \\
\hline RF (IU/L) & 15 & 133 & 12 & 66 & 38.3 & 125 & 0.387 \\
\hline ACCP (U/L) & 97 & 198.2 & 10 & 99 & 154 & 0.00 & $0.007^{\star}$ \\
\hline ESR (mm/hr) & 21 & 16 & 35 & 20 & 39 & 55 & 0.076 \\
\hline TNF-a (pg/ml) & 59 & 22.59 & 164.8 & 60.2 & 254.0 & 104.6 & \\
\hline TJ-28 & 12 & 19 & 6.00 & 3.75 & 12 & 5.00 & \\
\hline SJ-28 & 8.00 & 20 & 3.00 & 2.00 & 9.00 & 20 & \\
\hline HAQ-DI & 1.13 & 0.95 & 0.63 & 0.75 & 1.00 & 0.75 & $0.008^{\star}$ \\
\hline
\end{tabular}

TABLE 1: Comparison of metabolic, biochemical, and clinical parameters in groups of DAS28ESR.

* $\leq 0.05 p$-value.

${ }^{* *} \leq 0.001 \mathrm{p}$-value.

BMI: body mass index; DAS28-ESR: disease activity score of 28 joints; RF: rheumatoid factor; ACCP: anti-cyclic citrullinated peptide; ESR: erythrocyte sedimentation rate; TNF-a: tumor necrosis factor-alpha; TJ-28: tender joint count of 28 joints; SJ-28: swollen joint count of 28 joints; HAQ-DI: health assessment questionnaire disability index. 


\section{Cureus}

\begin{tabular}{|c|c|c|}
\hline Clinical, metabolic, and biomarker parameters & DAS28-ESR r(p) & TNF-a \\
\hline BMI $\left(\mathrm{kg} / \mathrm{m}^{2}\right)$ & $0.048(0.655)$ & $0.244\left(0.020^{*}\right)$ \\
\hline RF (IU/L) & $0.090(0.400)$ & $-0.109(0.306)$ \\
\hline ACCP (U/L) & $0.278\left(0.008^{*}\right)$ & $-0.027(0.804)$ \\
\hline $\mathrm{ESR}(\mathrm{mm} / \mathrm{hr})$ & $0.306\left(0.003^{*}\right)$ & $0.018(0.864)$ \\
\hline TNF-a (pg/ml) & $0.533(\leq 0.001)$ & 1.00 \\
\hline TJ-28 & $0.415(\leq 0.001)$ & $0.122(0.254)$ \\
\hline SJ-28 & $0.413(\leq 0.001)$ & $0.198(0.061)$ \\
\hline DAS28-ESR & 1.00 & $0.533(\leq 0.001)$ \\
\hline HAQ-DI & $0.194(0.067)$ & $0.005(0.964)$ \\
\hline
\end{tabular}

\section{TABLE 2: Correlation of DAS28-ESR and serum TNF- $\alpha$ with other variables.}

${ }^{*} \leq 0.05 p$-value.

${ }^{\star *} \leq 0.001 \mathrm{p}$-value.

BMI: body mass index; DAS28-ESR: disease activity score of 28 joints; RF: rheumatoid factor; ACCP: anti-cyclic citrullinated peptide; ESR: erythrocyte sedimentation rate; TNF-a: tumor necrosis factor-alpha; TJ-28: tender joint count of 28 joints; SJ-28: swollen joint count of 28 joints; $\mathrm{HAQ}-\mathrm{DI}$ : health assessment questionnaire disability index.

Multiple variable analysis was performed based upon the results of Spearman's correlation coefficient analysis. Our results showed no significant association between DAS28 and RF, ACCP, and ESR. Moreover, with every one-unit increase in serum TNF- $\alpha$, chances of disease activity will increase by $9.6 \%$ (Table 3).

\begin{tabular}{|c|c|c|c|c|c|c|}
\hline Outcome variable & Predictor variables & $\beta$ & Wald & Adjusted prevalence ratio & $95 \% \mathrm{Cl}$ & p-Value \\
\hline \multirow[t]{4}{*}{ DAS28 } & RF & 0.015 & 2.56 & 1.015 & $0.997-1.034$ & 0.109 \\
\hline & ACCP & 2.00 & 2.14 & 7.42 & $0.507-108.51$ & 0.143 \\
\hline & ESR & 0.026 & 0.018 & 1.027 & 0.991-1.064 & 0.145 \\
\hline & TNF-a & 0.096 & 8.365 & 1.101 & $1.031-1.175$ & $0.004^{\star}$ \\
\hline
\end{tabular}

TABLE 3: Multiple variable analysis with disease activity (DAS28-ESR).

\section{Discussion}

In our study, serum TNF- $\alpha$ was found to be significantly associated with DAS28-ESR in early RA patients. Patients with a low disease activity score had a significantly lower concentration of serum TNF- $\alpha$ than in the subgroups with moderate and high disease activity. Similar to our findings were the observations made by other studies conducted on newly diagnosed RA patients $[5,7,19]$. These findings show that TNF- $\alpha$ is expressed earlier in the pathologic events in DMARD naïve RA patients and can therefore be an important marker for the evaluation of disease activity. Previously in a study patients with high disease activity scores were reported to have significantly high concentrations of TNF- $\alpha$ in blood and synovial fluid as well [20]. However, in our study, synovial fluid samples were not taken.

The ACCP levels were higher in our study participants with severe DAS28 scores. This is maybe due to the high inflammatory activity in the majority of the patients positive for ACCP [21]. The values of TJ-28, the SJ28 , and HAQ-DI were higher in patients with severe disease activity. Associations of these variables with disease activity have also been reported in a previous study [22]. The reason for this can be the release of cytokines earlier in the course of RA that results in joint inflammation and pain, hence altering the functional ability in these patients [21]. BMI, RF, and ESR values showed no significant differences in the three groups of DAS28-ESR. 
Serum TNF- $\alpha$ was shown to have a positive correlation with DAS28 in our study participants. Previous studies also showed a positive correlation between plasma levels of TNF- $\alpha$ and activity of the disease in the newly diagnosed RA patients and not in patients who were on DMARD treatment [7,19]. Shrivastava et al. and others reported that DAS28 scores showed a significant correlation with serum TNF- $\alpha$ in the high disease activity subgroup of RA patients [7,23,24]. These findings suggest that TNF- $\alpha$ is a substantial mediator of inflammation in RA and plays a key role in the development and advancement of RA. However, Gheita et al. reported a significant negative correlation of the serum TNF- $\alpha$ level with the DAS28 in one genotype group and association between these two variables in the other genotype group of their study participants. A possible explanation for the varied associations between disease activity and serum TNF- $\alpha$ levels may be due to the TNF- $\alpha$ gene promoter polymorphism [25]. Our study showed a positive correlation of TNF- $\alpha$ with BMI. Similar findings were shown in a previous study [26]. The association between BMI with inflammation may be due to the increased levels of adipocytokines that act upon innate immune cells thus activating monocytes that produce increased levels of TNF- $\alpha$ [27]. Our study did not find any correlation of serum TNF- $\alpha$ levels with tender joint count and ESR in contrast to the association between TNF- $\alpha$ levels with TJ-28 and ESR in RA patients reported by Yen et al. [28].

A significant correlation of DAS28 with serum ACCP was also observed. The ACCP-containing immune complexes (ACCP-IC) triggers the release of inflammatory cytokine TNF $\alpha$ via the Fcy R-dependent pathway by macrophages [29]. These cytokines attack the synovium in joints of RA patients and lead to aggressive disease and ultimately results in joint erosion [21], hence, there is a more inflammatory activity in ACCP positive patients. Our study did not show a correlation between TNF- $\alpha$ and ACCP and RF titers. However, these variables correlated slightly but significantly in other studies [30].

Multivariable analyses revealed that high levels of serum TNF- $\alpha$ shows an increased probability of severity in disease activity in RA patients. However, Dissanayake et al. in their study observed a significant association of DAS28 with the cellular expression of TNF- $\alpha$ [4].

This research highlights not only the importance of TNF- $\alpha$ alpha in the severity of disease in early RA patients but can also contribute to the monitoring of disease activity patients on DMARD treatment. There were certain limitations to our study. It was a single-centered cross-sectional study and patients' samples were collected only once. They were not followed up after few months of treatment for RA. To validate our results, we propose that, multicenter studies, with a larger sample size and patient progress over a couple of months, are needed in selected RA patient groups.

\section{Conclusions}

Our study has found a significant correlation of TNF- $\alpha$ with DAS28-ESR in newly diagnosed RA patients. The available therapeutic agents that target this cytokine have excellent clinical effects in RA patients. In future studies on targeting TNF- $\alpha$ in the early stage of RA and its evaluation to monitor the progress of disease could be useful for patients undergoing anti-TNF therapy. If these patients are followed up properly, then serum TNF- $\alpha$ levels together with the assessment of clinical activity of disease by DAS28 could be used as a guide in determining the dose and intervals between dosing of TNF- $\alpha$ inhibitors in order to accomplish the desired therapeutic response. The number of patients in our study was relatively small, however, our findings are highly suggestive and require further large-scale investigations in the future to make use of serum TNF- $\alpha$ levels as a potential biomarker for evaluation of disease activity both before and after the start of anti-TNF therapy.

\section{Additional Information \\ Disclosures}

Human subjects: Consent was obtained or waived by all participants in this study. Ethical Review Committee of Ziauddin University issued approval (Ref code: 0920319MIPHY). The ERC Ziauddin University has reviewed this project in a meeting of March 2019 and gives the approval to conduct this study. Animal subjects: All authors have confirmed that this study did not involve animal subjects or tissue. Conflicts of interest: In compliance with the ICMJE uniform disclosure form, all authors declare the following: Payment/services info: All authors have declared that no financial support was received from any organization for the submitted work. Financial relationships: All authors have declared that they have no financial relationships at present or within the previous three years with any organizations that might have an interest in the submitted work. Other relationships: All authors have declared that there are no other relationships or activities that could appear to have influenced the submitted work.

\section{Acknowledgements}

We would like to thank Dr. Syed Mehfooz Alam (Consultant Rheumatologist). We also thank Moazzam Ali of the research lab, for his support in the laboratory work.

\section{References}

1. Brennan FM, McInnes IB: Evidence that cytokines play a role in rheumatoid arthritis . J Clin Invest. 2008, 
118:3537-45. 10.1172/JCI36389

2. Schett G, Gravallese E: Bone erosion in rheumatoid arthritis: mechanisms, diagnosis and treatment . Nat Rev Rheumatol. 2012, 8:656-64. 10.1038/nrrheum.2012.153

3. Alam J, Jantan I, Bukhari SN: Rheumatoid arthritis: recent advances on its etiology, role of cytokines and pharmacotherapy. Biomed Pharmacother. 2017, 92:615-33. 10.1016/j.biopha.2017.05.055

4. Dissanayake K, Jayasinghe C, Wanigasekara P, Sominanda A: Potential applicability of cytokines as biomarkers of disease activity in rheumatoid arthritis: Enzyme-linked immunosorbent spot assay-based evaluation of TNF- $\alpha$, IL-1 $\beta$, IL-10 and IL-17A. PLoS One. 2021, 16:e246111. 10.1371/journal.pone.0246111

5. Tetta C, Camussi G, Modena V, Di Vittorio C, Baglioni C: Tumour necrosis factor in serum and synovial fluid of patients with active and severe rheumatoid arthritis. Ann Rheum Dis. 1990, 49:665-7. 10.1136/ard.49.9.665

6. Gibofsky A: Overview of epidemiology, pathophysiology, and diagnosis of rheumatoid arthritis . Am J Manag Care. 2012, 18 (13 Suppl):S295-302.

7. Shrivastava AK, Singh HV, Raizada A, et al.: Inflammatory markers in patients with rheumatoid arthritis. Allergol Immunopathol (Madr). 2015, 43:81-7. 10.1016/j.aller.2013.11.003

8. Cunnane G, Fitzgerald O, Beeton C, Cawston TE, Bresnihan B: Early joint erosions and serum levels of matrix metalloproteinase 1 , matrix metalloproteinase 3 , and tissue inhibitor of metalloproteinases 1 in rheumatoid arthritis. Arthritis Rheum. 2001, 44:2263-74. 10.1002/1529-0131(200110)44:10<2263::aidart389>3.0.co;2-1

9. Hess A, Axmann R, Rech J, et al.: Blockade of TNF- $\alpha$ rapidly inhibits pain responses in the central nervous system. Proc Natl Acad Sci U S A. 2011, 108:3731-6. 10.1073/pnas.1011774108

10. Farrugia M, Baron B: The role of TNF- $\alpha$ in rheumatoid arthritis: a focus on regulatory T cells . J Clin Transl Res. 2016, 2:84-90.

11. Harre U, Schett G: Cellular and molecular pathways of structural damage in rheumatoid arthritis . Semin Immunopathol. 2017, 39:355-63. 10.1007/s00281-017-0634-0

12. Sikorska D, Kawka E, Rutkowski R, Samborski W, Witowski J: The intensity of joint pain in relation to changes in serum TNF $\alpha$ during therapy with anti-TNF $\alpha$ inhibitors. Inflammopharmacology. 2019, 27:67983. 10.1007/s10787-019-00564-X

13. Esalatmanesh K, Jamali R, Jamali A, Jamali B, Nikbakht M: Serum anti-cyclic citrullinated peptide antibodies may predict disease activity in rheumatoid arthritis. Rheumatol Int. 2012, 32:3799-805. 10.1007/s00296-011-2282-3

14. Aletaha D, Alasti F, Smolen JS: Rheumatoid factor, not antibodies against citrullinated proteins, is associated with baseline disease activity in rheumatoid arthritis clinical trials. Arthritis Res Ther. 2015, 17:229. 10.1186/s13075-015-0736-9

15. Marhadour T, Jousse-Joulin S, Chalès G, et al.: Reproducibility of joint swelling assessments in long-lasting rheumatoid arthritis: influence on Disease Activity Score-28 values (SEA-Repro study part I). J Rheumatol. 2010, 37:932-7. 10.3899/jheum.090879

16. Aletaha D, Neogi T, Silman AJ, et al.: 2010 Rheumatoid arthritis classification criteria: an American College of Rheumatology/European League Against Rheumatism collaborative initiative. Arthritis Rheum. 2010, 62:2569-81. 10.1002/art.27584

17. The Health Assessment Questionnaire@ Stanford University School of Medicine Division of Immunology and Rheumatology. (2001). Accessed: January 19, 2021: http://www.chcr.brown.edu/pcoc/EHAQDESCRSCORINGHAQ372.PDF.

18. DAS28 - disease activity score calculator for rheumatoid arthritis [updated] . (2020). Accessed: August 10, 2020: http://www.4s-dawn.com/DAS28/.

19. Samimi Z, Kardideh B, Chalabi M, Zafari P, Taghadosi M: The plasma Tumor Necrosis Factor- $\alpha$ (TNF- $\alpha$ ) does not have any correlation with disease activity in rheumatoid arthritis patients treated with disease modifying anti-rheumatic drugs (DMARDs). Braz J Pharm Sci. 2020, 56:1-7. 10.1590/s217597902019000418551

20. Petrović-Rackov L: [Evaluation of the degree of clinical rheumatoid arthritis activity based on the concentrations of cytokines TNF-alpha, IL-12, IL-15, and IL-18 in serum and synovial fluid]. Voinosanit Pregl. 2006, 63:21-6. 10.2298/vsp0601021p

21. Seegobin SD, Ma MH, Dahanayake C, Cope AP, Scott DL, Lewis CM, Scott IC: ACPA-positive and ACPAnegative rheumatoid arthritis differ in their requirements for combination DMARDs and corticosteroids: secondary analysis of a randomized controlled trial. Arthritis Res Ther. 2014, 16:R13. 10.1186/ar4439

22. Ghosh A, Ghosh B, Pain S, et al.: Comparison between DAS28, CDAI and HAQ-DI as tools to monitor early rheumatoid arthritis patients in eastern India. Indian J Rheumatol. 2011, 6:116-22. 10.1016/S09733698(11)60073-3

23. Meyer PW, Hodkinson B, Ally M, et al.: Circulating cytokine profiles and their relationships with autoantibodies, acute phase reactants, and disease activity in patients with rheumatoid arthritis. Mediators Inflamm. 2010, 2010:158514. 10.1155/2010/158514

24. Osiri M, Wongpiyabovorn J, Sattayasomboon $\mathrm{Y}$, Thammacharoenrach $\mathrm{N}$ : Inflammatory cytokine levels, disease activity, and function of patients with rheumatoid arthritis treated with combined conventional disease-modifying antirheumatic drugs or biologics. Clin Rheumatol. 2010, 35:1673-81. 10.1007/s10067016-3306-x

25. Gheita TA, Azkalany GS, Gaber W, Mohey A: Clinical significance of serum TNF $\alpha$ and-308 G/A promoter polymorphism in rheumatoid arthritis. Egypt Rheumatol. 2015, 37:49-54. 10.1016/j.ejr.2014.07.001

26. Olszanecka-Glinianowicz M, Zahorska-Markiewicz B, Janowska J, Zurakowski A: Serum concentrations of nitric oxide, tumor necrosis factor (TNF)-alpha and TNF soluble receptors in women with overweight and obesity. Metabolism. 2004, 53:1268-73. 10.1016/j.metabol.2004.07.001

27. George MD, Baker JF: The obesity epidemic and consequences for rheumatoid arthritis care . Curr Rheumatol Rep. 2016, 18:6. 10.1007/s11926-015-0550-z

28. Yen J, Chen J, Tsai W, Liu H: Correlation of tumor necrosis factor alpha levels with disease activity of rheumatoid arthritis. Chin J Microbiol Immunol. 1992, 25:232-43. 


\section{Cureus}

29. Laurent L, Clavel C, Lemaire O, et al.: Fcy receptor profile of monocytes and macrophages from rheumatoid arthritis patients and their response to immune complexes formed with autoantibodies to citrullinated proteins. Ann Rheum Dis. 2011, 70:1052-9. 10.1136/ard.2010.142091

30. Takeuchi T, Miyasaka N, Inui T, Yano T, Yoshinari T, Abe T, Koike T: High titers of both rheumatoid factor and anti-CCP antibodies at baseline in patients with rheumatoid arthritis are associated with increased circulating baseline TNF level, low drug levels, and reduced clinical responses: a post hoc analysis of the RISING study. Arthritis Res Ther. 2017, 19:194. 10.1186/s13075-017-1401-2 